\title{
A reforma psiquiátrica e seus desdobramentos: representações sociais dos profissionais e usuários da atenção psicossocial
}

\author{
Psychiatric reform and their developments: social representations of professionals and users \\ of the psychosocial care \\ Juliana Pessoa Costa ${ }^{1}$ \\ Maria Salete Bessa Jorge ${ }^{2}$ \\ Maria da Penha de Lima Coutinho ${ }^{3}$ \\ Edmara Chaves Costa ${ }^{4}$ \\ Ítala Thaise Aguiar Holanda ${ }^{5}$
}

\begin{abstract}
RESUMO: Esta pesquisa objetivou apreender as representações sociais de usuários e profissionais de saúde sobre a reforma psiquiátrica. Participaram 50 usuários e 30 profissionais da saúde do Centro de Atenção Psicossocial, Centro de Atenção Psicossocial Álcool e outras Drogas e da Estratégia Saúde da Famíliada cidade de Fortaleza-CE, que foram submetidos a Técnica de Associação Livre de Palavras, com os estímulos indutores: loucura, desinstitucionalização, internação psiquiátrica e reforma psiquiátrica. Os dados foram analisados pela análise fatorial de correspondência e os resultadosapontaram para uma aproximação entre o conhecimento do senso comum e o conhecimento erudito quando utilizadas as associações ao estímulo reforma psiquiátrica, ambos consideraram o processo de humanização eluta, além de se remeter a utopia, caps, melhor e acreditar. A loucura é objetivada como sinônimo de doença mental que provoca crises e tem como consequências: sofrimento, desespero e preconceito. A desinstitucionalização, bem como a reforma psiquiátrica, foi representada como ação progressiva de liberdade e inserção, voltada para a desconstrução do modelo asilar e expansão de uma rede de atenção extra-hospitalar. E a internação psiquiatrica como uma forma de exclusão do doente mental. Os resultados também evidenciaram a importância do suporte familiar e social no contexto da saúde mental. Espera-se que esses resultados venham a contribuir para uma melhor conscientização acerca deste fenômeno.
\end{abstract}

Palavras-chave: saúde mental; reforma psiquiátrica; representações sociais; serviços de saúde mental; reabilitação.

ABSTRACT: This research aimed to understand the social representations of service users and healthcare professionals about psychiatric reform. The participants were 50 users and 30 professionals from the Psychosocial Care Center, Psychosocial Care Center Alcohol and Other Drugs and Family Health Strategy in the city of Fortaleza-CE, who underwent Free Association Technique of Words, with stimuli: madness, deinstitutionalization, psychiatric hospitalization and psychiatric reform. Data was analyzed by factorial correspondence analysis, the results showed that the common sense knowledge about the psychiatric reform using the associations of stimuli already mentioned like the erudite knowledge, considered this as a process of humanization, struggle, utopia, caps, better and believe. Madness as mental illness that causes crisis and its consequences: suffering, despair and prejudice. The deinstitutionalization and reform were represented as the process of change toward the deconstruction of the model shelter and

\footnotetext{
1 Mestre em Saúde Coletiva pela Universidade Estadual do Ceará (UECE) - Fortaleza, CE, Brasil. E-mail: julianapessoato@hotmail.com.

2 Pós-doutora em Enfermagem pela Universidade de São Paulo (USP); Professora Titular da Universidade Estadual do Ceará (UECE) - Fortaleza, CE, Brasil.

${ }^{3}$ Pós- doutora pela Universidade Aberta de Lisboa; Professora da Universidade Federal da Paraíba - João Pessoa, PB, Brasil.

4 Pós-doutora em Biotecnologia pela Universidade Estadual do Ceará (UECE); Professora Adjunto I da Universidade da Integração Internacional da Lusofonia Afro-Brasileira (Unilab) - Fortaleza, CE, Brasil.

${ }^{5}$ Mestre em Saúde Coletiva pela Universidade Estadual do Ceará (UECE) - Fortaleza, CE, Brasil.
} 
expansion of a network of a out hospital care. In addition, the psychiatric hospitalization as a form of mental ill exclusion. The results also showed the importance of family and social support in the context of mental health. It is expected that these results could contribute to a better consciousness of this phenomenon.

Keywords: mental health; psychiatric reform; social representations; mental health services; rehabilitation.

\section{Introdução}

A história da reforma psiquiátrica brasileira é um processo em construção, compreendida como a desconstrução de saberes, discursos e práticas psiquiátricas, relativos aos manicômios. A partir da década de 1970, essa temáticapassou a ser amplamente questionada e discutida, pois o modelo adotado no hospital psiquiátrico era marcado por sua assistência hospitalocêntrica que se caracterizava por ser mais alienadora do que produtora de ressocialização e reabilitação (Alves \& Oliveira, 2010).

Essa situação se modifica a partir da década de oitenta com o início da luta antimanicomial no Brasil, instaurada durante o Congresso de Trabalhadores de Saúde Mental realizado na cidade de Bauru, em São Paulo, no ano de 1987. Essas discussões resultaram no projeto de leique propunha a reforma psiquiátrica no país, de autoria do deputado Paulo Delgado, e entrou em tramitação no Congresso Nacional em 1989. Em 6 de abril de 2001, foi sancionada a Lei Federal no 10.216, Lei Paulo Delgado, que instituiu a reforma psiquiátrica e a substituição dos modelos institucionalizados no tratamento do portador de sofrimento psíquico (Ministério da Saúde, 2005).

Novas formas de cuidado em saúde mental são propostas, sustentadas no modo psicossocial, com ações centradas não mais na doença, mas no indivíduo e nas suas potencialidades. O novo modelo, denominado de assistencial, propõe-se um conjunto de ações inspiradas na substituição do modelo manicomial dos hospitais psiquiátricos pela criação de uma rede de serviços territoriais de atenção psicossocial, dispositivos institucionais de características não asilares, alternativas de base comunitária. Não pretendeabolir o tratamento clínico, mas eliminar a prática do internamento como uma forma de exclusão social. Para isto, preconiza o atendimento por equipe multiprofissional, com atuação interdisciplinar, no sentido de fortalecer a reintegração sociocultural das pessoas com transtorno mental (Saraceno, 1999; Basaglia, 2005; Nasi \& Schneider, 2011).

Sob a égide desses pressupostos, foi aprovada a Portaria $n^{\circ}$ 336/2002 do Ministério da Saúde, preconizando essas novas configurações de prestação do cuidado em saúde mental, com criaçãoe disponibilização de serviços pertencentes ao Sistema Único de Saúde (SUS), tais como:Centros de Atenção Psicossocial (CAPS), leitos psiquiátricos em Hospitais gerais, ambulatórios, Unidades Básicas de Saúde (UBS), Serviços Residenciais Terapêuticos (SRTs) e redes de apoio (associações de moradores de bairros, igrejas, grupos de ajuda mútua, escolas e universidades), com a finalidade de atender pessoas que apresentam sofrimento psíquico, como transtornos mentais, psicoses, neuroses graves, transtornos relacionados às substâncias psicoativas e que necessitem de cuidado intensivo (Pitta, 2011; Brasil, 2004).

Dentre os serviços, o CAPS se destaca comopromotorda articulação entre os cuidados clínicos e os programas de reabilitação psicossocial, por meio de trabalhos que visem à inserção social, com a construção de vínculos e interação humana, sempre com respeito às potencialidades e às limitações individuais e aos princípios de cidadania, estimulando o 
protagonismo de cada usuário perante sua vida (Ministério da Saúde, 2005; Antunes \& Queiroz, 2007).

Esse novo contexto de cuidado em saúde mental possibilita o surgimento, no Brasil, da ideia de desinstitucionalização, que consiste não apenas em retirar os sujeitos de dentro dos hospitais psiquiátricos, mas reinseri-losem suas comunidades, fortalecendo seus vínculos e desenvolvendo sua autonomia e potencialidade (Santos, 2000). Assim, propicia, com originalidade, o fortalecimento de uma sociedade fundamentada no exercício pleno da cidadania, da liberdade, da justiça social e no respeito aos diferentes, entre outros princípios, possibilitando a reconstrução de vítimas de um sistema, na maioria das vezes, oportunista e opressor (Boarini, 2000; Rotelli, 2001).

Neste sentido, o processo de desinstitucionalização em saúde mental considera os profissionais como sujeitos inseridos nos serviços de saúde, responsáveis por favorecer formas de cuidados condizentes com a Reforma Psiquiátrica, e os usuários como portadores de um saber construído da vivencia cotidiana (Jodelet, 2001).

Apesar desse direcionamento inovador, o atual conceito de saúde mental ainda é alicerçado nos aspectos socioculturais da loucura, construto este baseado no estereótipo da loucuraalicerçada nos elementos da violência e da irracionalidade, foco de repulsa, isolamento e visões estigmatizadoras do transtorno mental (Ribeiro, 2015).

Diante do exposto, surgiu o interesse em investigar quais os pensamentos, opiniões, crenças, esteriótipos, atitudes, preconceitos que os grupos de pertença de usuários e profissionais de saúde possuiemsobre a reforma psiquiátrica, bem como investigar as representações associadas ao processo atual de desinstitucionalização e reforma psiquiátrica tanto no lócus especializado da saúde mental (CAPS) quanto na rede básica de saúde (ESF).

Em face dessas premissas, este artigo objetivou apreender as representações sociais de usuários e profissionais de saúde dos serviços do Centro de Atenção Psicossocial (CAPS Geral), Centro de Atenção Psicossocial Álcool e outras Drogas (CAPS AD) e da Estratégia Saúde da Família (ESF) acerca da reforma psiquiátrica e do processo de desinstitucionalização, além de investigar as RS da loucura e da internação psiquiátrica no atual contexto de saúde. Nesse sentido, utilizou-se como eixo norteador a abordagem psicossocial da Teoria das Representação sociais (TRS), como possibilidade de compreender como estes participantes constroem um conhecimento prático.

Segundo Moscovici (2001, 2003), as Representações Sociais (RS) são conjuntos simbólicos/práticos/dinâmicos cujo status é o de uma produção e não de uma reprodução. Essa assertiva significa que a RS não é uma simples reação a estímulos exteriores, mas a escolha e a utilização, a partir de repertório circulante na sociedade, de informações destinadas à interpretação e a elaboração do real. Considera, ainda, que toda informação nova que chega ao sujeito, inicialmente, é confrontada com as informações que este já possui, e a partir dessa confrontação são incorporadas em um sistema particular de categorias denominado esse processo de ancoragem, que por sua vez é um dos processos de formação das representações sociais.

Desse modo, ter acesso as RS de um objeto social é tentar compreender as formas que as pessoas utlilizam para criar, transformar e interpretar uma temática vinculada a sua realidade, assim como, identificar seus pensamentos, sentimentos, percepções e experiencias de vida compartilhadas, de acordo com a classe social a que pertencem e às instituições as quais pertencem (Coutinho, 2005). 
No campo da saúde mental, observam-se vários estudos inerentes a esse fenômeno (Jodelet, 2005; Antunes \& Queiroz, 2007; Nasi \& Schneider, 2011; Cândido et al., 2012; Franken, Coutinho \& Ramos, 2012; Paulon et al., 2013). No entanto, faz-se necessário avançar, principalmente no que tange a reforma psiquiátrica, considerando as mudanças paradigmáticas que vem ocorrendo desde a década de 60, implicando reestruturação de saberes e práticas, especialmente, por parte dos profissionais de saúde.

Dentre os conhecimentos científicos e aqueles produzidos pelo senso comum sobre a reforma psiquiátrica indaga-se sobre: quais as representações sociais dos profissionais de saúde e usuários dos serviços da atenção psicossocial sobre a reforma psiquiátrica? Essa questão, que norteou a presente pesquisa, é relevante, tendo em vista o significativo papel atribuído a atenção em saúde mental realizada pelos Centros de Atenção Psicossocial (CAPS), mas também pela expectativa em torno da função de apoio matricial em saúde mental assumido pela ESF. Assim sendo, pode-se buscar e refletir sobre as dificuldades, estratégias e perspectivas quanto as transformações advindas da reforma psiquiátrica e as relações sociais tecidas sobre a loucura e o antagonismo entre internação psiquiátrica e desinstitucionalização.

\section{Método}

A pesquisa foi realizada no município de Fortaleza, capital do estado do Ceará, nordeste do Brasil, mais precisamente naSecretaria Executiva Regional V (SER V). Os participantes da pesquisa foram os usuários e profissionais dos Centros de Atenção Psicossocial (CAPS Geral e AD) e da Estratégia Saúde da Família (ESF).

A pesquisa foi realizada com 80 participantes, sendo 50 usuários com transtornos mentais e dependentes químicos e 30 profissionais de saúde, entre eles médicos, enfermeiros, psicólogos, assistentes sociais, farmacêuticos e terapeutas ocupacionais dos CAPS Geral, CAPS AD e ESF.

Antes da realização do trabalho de campo, o estudo foi submetido à análise do Comitê de Ética em Pesquisa com Seres Humanos (CEP) da Universidade Estadual do Ceará (UECE) recebendo parecer favorável sob no 681.467. Desse modo, seguiram-se os princípios da bioética descritos na Resolução 466/12 do Conselho Nacional de Saúde (CNS) (CNS, 2012).

Inicialmente, os participantes assinaram o Termo de Consentimento Livre e Esclarecido, autorizando sua participação na pesquisa, em seguida responderam a Técnica de Associação Livre de Palavras (TALP). Ela é um instrumento que permite a atualização de elementos implícitos ou latentes que seriam perdidos ou mascarados nas produções discursivas. É uma técnica projetiva amplamente utilizada no âmbito da Psicologia Social, principalmente quando se trabalha com o aporte teórico das RS, o qual permite evidenciar universos semânticos que refletem os universos comuns de palavras em face de diferentes estímulos e sujeitos ou grupos (Nóbrega \& Coutinho, 2011).

$\mathrm{Na}$ TALP, foram utilizados quatro estímulos indutores: "Loucura", "Desinstitucionalização", "Internação Psiquiátrica" e "Reforma Psiquiátrica". Os dados advindos deste instrumento foram processados pelo software Tri-deux-mots (versão 2.2), o qual realizou as cinco etapas para o processamento de dados: IMPMOT (gerando o dicionário e a frequência absoluta), TABMOT (tabulação dos dados), ECAPEM (construção dos fatores), ANECAR (gera o arquivo com as informações da análise fatorial de 
correspondência) e PLANFA (a produção do gráfico com as coordenadas ou fatores) (Cibois, 1995).

\section{Resultados}

Fizeram parte desta pesquisa 80 participantes, dos quais 50 eram usuários dos serviços de saúde, sendo $66 \%$ do sexo feminino. Quanto ao serviço de origem, 25 usuários eram do CAPS Geral, 15 do CAPS AD e 10 da ESF. No que se refere a idade, pôde-se verificar que $16 \%$ estavam na faixa etária de 20 a 28 anos; $40 \%$ de 29 a 36 anos; $26 \%$ de 37 a 44 anos; e $18 \%$, acima de 45 anos.Quanto a escolaridade, $38 \%$ tinham ensino médio incompleto e $28 \%$ apresentavam ensino fundamental incompleto.

Entre os 30 profissionais de saúde, fizeram parte da pesquisa nove enfermeiros, oito assistentes sociais, cinco farmacêuticos, três médicos, dois dentistas, além de um músico, um psicólogo e um terapeuta ocupacional. A maioria dos profissionais era do sexo feminino 90\%, sendo 13 profissionais do CAPS Geral, 10 do CAPS AD e 7 da ESF. Quanto a faixa de idade, três profissionais tinham de 20 a 28 anos; $56,6 \%$, de 29 a 36 anos e 33,3\%, de 37 a 44 anos.

Em relação às representações sociais advindas da TALP, apresentadas na Figura 1, elas propiciam uma leitura representacional das variações semânticas associadas aos estímulos indutores: "Loucura", "Desinstitucionalização", "Internação Psiquiátrica" e "Reforma Psiquiátrica", e as elaboradas por usuários e profissionais da área da saúde revelando as aproximações e os distanciamentos das modalidades de construção dos eixos ou fatores (F1 e F2). O somatório desses dois fatores evidenciou um poder explicativo de $62,4 \%$ da

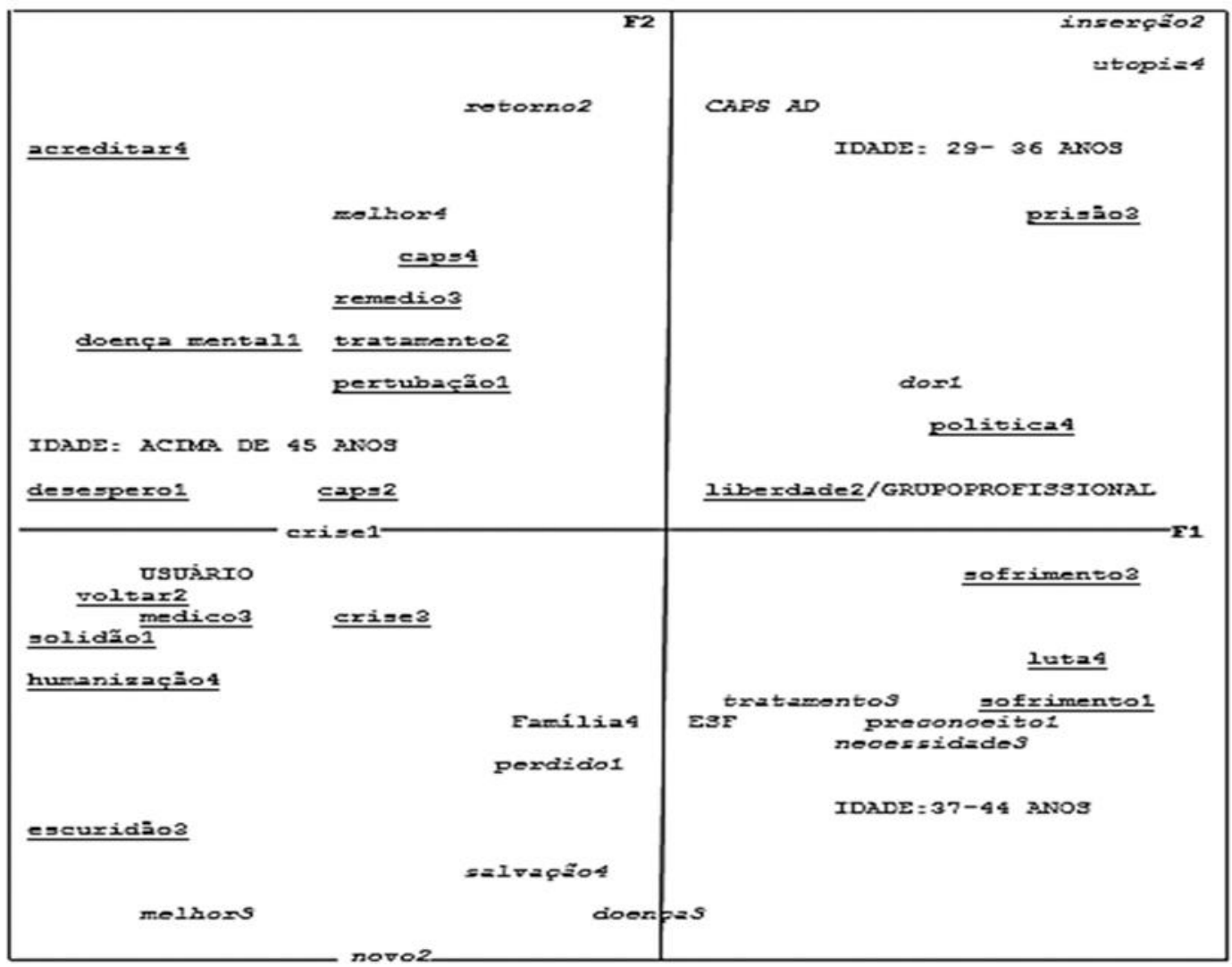

Figura 1 - Plano fatorial de correspondência das representações sociais acerca da reforma psiquiátrica elaboradas por usuários e profissionais do CAPS Geral, CAPS AD e ESF. Legenda: Fator 1(F1), localiza-se no eixo horizontal à direita e à esquerda, com palavras sublinhadas; Fator 2(F2), localiza-se no eixo vertical superior e inferior, com 
palavras em itálico. Variáveis fixas: em letras maiúsculas. Estímulos indutores: 1= Loucura, 2= Desinstitucionalização, 3= Internação Psiquiátrica e 4= Reforma Psiquiátrica.

variância total das respostas, sendo que o $F 1$ apresentou 44,8\%, e o F2, 17,6\%, demonstrando, parâmetros estatísticos com consistência interna e fidedignidade, tendo em vista pesquisas realizadas no âmbito das RS (Nóbrega \& Coutinho, 2011).

No fator 1 (F1) do lado esquerdo foram observadas as construções dos participantes usuários com idades acima dos 45 anos, em relação ao estímulo indutor 1 - "Loucura", este foi objetivado em: crise, desespero, solidão, perturbação e doença da mental. Em relação ao estímulo 2 - "Desinstitucionalização", observou-se as seguintes objetivações: caps, voltar etratamento. Para o terceiro estímulo - "Internação Psiquiátrica", os participantes objetivaram em crise, remédio e médico. O último estímulo - "Reforma Psiquiátrica" - foi objetivado emcaps, humanização e acreditar.

Ainda no F1, do lado direito encontra-se o campo semântico elaborado pelos profissionais da saúde que objetivaram a "Loucura" em sofrimento. Para o estímulo "Desinstitucionalização" foi observada a evocação de liberdade. O terceiro estímulo "Internação Psiquiátrica", este foi objetivado em sofrimento e prisão, e a "Reforma Psiquiátrica" como luta e política.

Em relacao ao fator (F2), eixo vertical superior, encontram-se os pensamentos dos participantes do CAPS AD com idade entre 29 e 36 anos. Para o primeiro estímulo, consta no plano fatorial a evocação de loucura como dor. O estímulo 2 ("Desinstitucionalização") foi objetivado como uma inserção, um retorno. Para o estímulo"Internação Psiquiátrica"não houve contribuição significativa, portanto, não foram observadas evocações. Já a "Reforma Psiquiátrica" foi vista como uma utopia e como melhor.

Neste mesmo eixo no lado inferior, encontram-se os campos semânticos dos representantes daESF, com idades entre 37 e 44 anos, que objetivaram a "Loucura" como preconceito e perdido, em relação a "Desinstitucionalização", esta foi vista como novo. 0 estímulo 3 "Internação Psiquiátrica" foi objetivado em tratamento, necessidade, doença e melhor. Quanto ao último estímulo, "Reforma Psiquiátrica", os participantes a consideraram como família e salvação.

\section{Discussão}

Diante dos resultados apresentados, pôde-se verificar que, de modo geral, os participantes representaram a "Loucura" como sinônimo de doença mental que provoca perturbação e crises, tendo como consequências para os usuários: desespero e solidão; para os participantes dos serviços de atenção psicossocial a objetividade no sofrimento e na dor; e como perdido e preconceitopara os da ESF. Nesta construção percebe-se quea loucura é representada no conhecimento prático, no qual a doença mental é vista como um problema relativo ao mau funcionamento ou perturbação para os usuários e como uma questão psicoafetiva para os profissionais. Essas inferências se aproximam ao que Foucault (2010) preconiza sobre a doença mental, quando o autor faz menção a obscuridade e ausência consensual de uma conceituação.

Dentre as palavras evocadas no primeiro estímulo, predominaram as palavras: sofrimento/dor (CAPS Geral e AD) e perdido/preconceito (ESF). Infere-se por meio dessas associações que esses participantes, dependendo do serviço de origem, relacionamo padrão 
de anormalidade associado à loucura, considerando como foco o sujeito que sofre oucomoprocesso de exclusão e estigmas inerentes aoprocesso de adoecimento.Nesse sentido, Lopes (2013) aponta que os profissionais percebem os pacientes como pessoas que precisam de ajuda, por estarem vivenciando uma experiência de adoecimento psíquico. Além disso, são percebidas como pessoas que podem despertar julgamentos estereotipados ecompaixão.

Conforme reforça Cândido et al. (2012) "para haver mudanças nos cenários tradicionais decuidado em saúde mental é necessário, primeiramente, que sejam conhecidas as âncoras do preconceito, bemcomo quem são os seus atores contribuintes" que podem ser uma expressão do senso comum partilhado socialmente na comunidade na qual está inserido o usuárioou mesmo preencher o imaginário de gestores e profissionais de saúde.

Observou-se que quanto a"Desinstitucionalização" o conhecimento prático emerge de forma associada ao CAPS, em que o tratamento éefetivado indissociável do contexto social onde vive o sujeito, em vez de um ambiente institucional, o que pode explicar a evocação de voltar. Vale registar que este processo de desinstitucionalização surgiu da necessidade de mudar o sistema psiquiátrico centrado na assistência hospitalar com a finalidade dedesconstrução de saberes e práticas psiquiátricas, perspectiva que fundamenta o movimento de reforma psiquiátrica e a política de saúde mental brasileira.

Pode-se observar uma representação congruente dos participantes usuários e profissionais da saúde do CAPS Geral e CAPS AD, em ambos os eixos, e ligada a reinserção social e serviços substitutivos, expressos como voltar/tratamento, liberdade e retorno/inserção, respectivamente. Contudo, os participantes da ESF objetivaram a desinstitucionalização como algo novo. Deve-se atentar ao fato de que saúde mental não consta do perfil de prioridades regulamentadas pela Portaria $n^{\circ} 648 / G M(2006)$ da ESF, apesar dos problemas psíquicos não estarem desvinculados dos demais enfrentados pelas equipes de Saúde da Família, incluindo depressão, alcoolismo e suicídio. E apenas há alguns anos tem-se implementado iniciativas de agregar ações de saúde mental na ESF no Brasil (Paulon et al., 2013).

A desinstitucionalização está atrelada ao tratamento que por sua vez está ligado ao CAPS, na construção representacional dos usuárioscomo nova forma de substituição do modelo manicomial que se apresenta como fortalecimento de uma rede de serviços substitutivos. Desta maneira, os Centros de Atenção Psicossociais (CAPS) são serviços de atenção diária em saúde mental, que busca consolidar práticas que favoreçam ações promotoras de emancipação e melhoria na qualidade de vida das pessoas, daí a objetivação com evocações de voltar/retorno, liberdade e inserção (Bedin \& Scarparo, 2011).

Nesse contexto, a desinstitucionalização passou por mudanças paradigmáticas uma vez que anteriormente o tratamento eracentrado apenas na instituição. Em consequência das políticas sociais e de intervenções legislativas, foi sancionada a crise da centralidade do manicômio; o que representou um grande avanço no processo de reforma (Venturini,1999). Esta também foi uma forma de re-inserção do doente mental a sociedade como um todo e, principalmente, para a família.

A desinstitucionalização como processo de quebra de paradigma visa a desconstrução do modelo asilar, procurando expandir e consolidar uma rede de atenção extra-hospitalar, de modo a atender as demandas territoriais específicas sem desassistir e indo além da pura desospitalização (Hirdes, 2009). Dessa forma, o CAPS deve proporcionar um tratamento que 
visa os cuidados de reinserção social de seus usuários por meio do acesso ao lazer, retorno ao lar, trabalho e reconstrução dos laços comunitários e familiares (Lopes, 2013).

Quanto ao campo semântico "Internação Psiquiátrica", os usuários apresentaram em crise, remédio e médico ancorada no modelo biomédico. Percebe-se nesses discursos associados a questão da internação pautada no modelo de assistência hospitalocêntrica que se caracterizava principalmente pela exclusão dos pacientes do seu entorno social (Alves \& Oliveira, 2010), mas registra-se essa ancoragem dada a eventos extremos envolvendo, provavelmente, pessoas em situações de crise associada a transtornos severos e graves. Para Ferigato, Campos e Ballarin (2007), a introdução do termo crise aborda as consequências psicológicas causadas nos seres humanos após tragédias ou catástrofes, uma concepção que atribui o adoecer psíquico, a solidão e causas externas ou sociais.

Por sua vez, os profissionais evocaram as expressões sofrimento e prisão para o estímulo indutor internação psiquiatrica. Nesse contexto, as respostas podem ser traduzidas como o sofrimento e o aprisionamentoque as pessoas com transtorno mental foram submetidas ao longo da história, a complexidade da compreensão da doença, a exclusão e o confinamento em hospitais psiquiátricos, de modo que por muito tempo permaneceram em longas internações, excluídos e marginalizados. Desse modo, as RS objetivadas na instituição enquanto "sofrimento e aprisionamento" e ancoradas na esfera psicossocial, demonstram a vulnerabilidade pessoal, desordem social, privação de liberdade e causas do sofrimento de ordem física e psicológica da pessoa com transtorno mental (Jodelet, 2001).

Por outro lado, os participantes da ESF expressaram evocações de doenças, tratamento, necessidade e melhor para "Internação Psiquiátrica", o que vai de encontro aos achados de Paulon et al. (2013) segundo o qual as concepções na rede básica de atenção "ainda são muito próximas do modelo psiquiátrico tradicional, (...) sendo a mediação e a internação as respostas terapêuticas mais aceitas" (p. 28). Vale salientar queas internações psiquiátricas têm como objetivo tratar, cuidar e proteger 0 doente mental de comportamentos perigosos. A institucionalização é indicada em casos graves, no esgotamento dos serviços extra-hospitalares (Gonçalves, Vieira \& Delgado, 2012).

A "Reforma Psiquiátrica" foi objetivada em humanização, acreditar e caps pelos usuários e em luta e política, pelos profissionaisambos ancorados na esfera social. Provavelmente, a questão da humanização encontra-se mais associada a pessoa que precisa ser vista e tratada com mais respeito e dignidade; o reconhecimento da importância do processo de reforma expresso em acreditar e das instituições no caso de caps. Enquanto a luta e a políticaestão mais ligadas à reforma enquanto processo de construção e consolidação.

Percebe-se então, que o processo da Reforma Psiquiátrica e o surgimento dos serviços substitutivos favoreceram a construção da interação social, construção da cidadania e autonomia, quando se objetiva a reforma psiquiátrica como família e salvação pelos participantes da ESF. O sujeito inserido nesses serviços torna-se participante de seu tratamento, existe trabalho com a participação da família, espaço para o momento da fala, escuta e inclusão social. Por outro lado, tendo na Política de Saúde Mental um processo de transformação da relação sociedade e loucura, buscando construir novos rumos para a inclusão social, conforme evidenciado nas evocações de utopia como modelo ideal de reforma e melhor pelos participantes do CAPS AD (Bedin\& Scarparo, 2011). 


\section{Conclusão}

No presente estudo, as representações dos profissionais de saúde e dos usuários dos serviços da CAPS e demais orgãos mencionados acerca da reforma psiquiátricarevelam pensamentos consensuais, mas também dissensos. Consensuais quando estes remetem a reforma como um processo de luta por serviços substitutivos que favoreçam a construção da interação social, construção da cidadania e autonomia.E dissensos relacionados a ESF, que merecem destaque, enquanto reconhecimento da relevância da atenção básica no apoio matricial à saúde mental.

Conforme pôde-se observar pelos resultados advindos das associações dos participantes, as representações sociais da reforma psiquiátrica encontram-se imbricadasem um universo complexo que envolve aspectos: culturais, sociais e econômicos. Apreendê-las proporciona o conhecimento de seus pressupostos teóricos e práticoscom a realidade do cuidado em saúde mental no contexto atual.

O CAPS aparece como serviço de reabilitação psicossocial que valoriza o contextosocial, a ressocialização do indivíduo e a participação da família como suporte fundamentalno tratamento. Outro aspecto relevante é o preconceito que a pessoa com transtorno mental enfrenta no seu cotidiano e a dificuldade na inserção do mercado de trabalho.

Apresenta-se como limitação deste estudo a utilização de uma amostra não probabilística, uma vez que esta não permite a possibilidade de generalização dos resultados. Dessa forma, sugere-se cautela ao comparar estes resultados aos de outros autores.Destaca-se também, a aplicação de um único instrumento para a apreensão das representações sociais do fenômeno estudado.

Contudo, espera-se que os resultados contribuam para uma melhor compreensão da reforma psiquiátrica como processo de luta ainda presente na atual conjuntura e contribua também para um olhar mais humanizado no que tange a doença mental e do ser acometido por esta. Espera-se que este estudo sirva de motivação para outros pesquisadores na busca de novas pesquisa utilizando-se de uma maior amplitude amostral e outros instrumentos que venham possibilitar maior visibilidade sobre a temática.

\section{Referências}

Alves, M., \& Oliveira, R. M. P. (2010). Enfermagem psiquiátrica: discursando o ideal e praticando o real. Escola Anna Nery Revista de Enfermagem, 14(1), 64-70. Recuperado em 18 dezembro, 2015, de http://www.scielo.br/pdf/ean/v14n1/v14n1a10.pdf.

Antunes, S. M. M. de O., \& Queiroz, M. (2007). A configuração da reforma psiquiátrica em contexto local no Brasil: uma análise qualitativa. Cad. Saúde Pública, Rio de Janeiro, 23(1), p. 207-215. Recuperado em 10 março, 2016, de http://www.scielo.br/scielo.php?script=sci_arttext\&pid=S0102-311X2007000100022.

Basaglia, F. (2005). Escritos selecionados em saúde mental e reforma psiquiátrica. In P. Amarante (Org.). (J. A. d’Ávila Melo, Trad.). Rio de Janeiro: Garamond.

Brasil. Ministério da Saúde. (2004). Legislação em saúde mental: 1990-2004. Ministério da Saúde, SecretariaExecutiva, Secretaria de Atenção à Saúde (5. ed. ampl.). Brasília: Ministério da Saúde.

Bedin, M. D., \& Scarparo, H. B. K. (2011). Integralidade e saúde mental no SUS a luz da teoria da complexidade de Edgar Morin. Psicologia: Teoria e Prática, 13(2), 195-208. Recuperado em 18 fevereiro, 2014, de http://pepsic.bvsalud.org/pdf/ptp/v13n2/v13n2a15.pdf. 
Boarini, M. L. (org.). (2000). Desafios na atenção à saúde mental. Maringá: Eridem.

Cândido, M. R., Oliveira, E. A. R., Monteiro, C. F. S., Costa, J. R., Benício, G. S. R., \& Costa, F. L. L. (2012). Conceitos e preconceitos sobre transtornos mentais: um debate necessário. Rev. Eletrônica Saúde Mental Álcool Drog., 8(3), 110-117. Recuperado em 14 março, 2016, de http://pepsic.bvsalud.org/scielo.php?script=sci_arttext\&pid=S1806-69762012000300002\&Ing $=$ pt\&tlng=en.

Cibois, U. F. R. (1995). Tri-deux-mots. Versão 2.2 Paris: Sciences Sociales.

Conselho Nacional de Saúde (2012). Resolução no 466, de 12 de dezembro de 2012. Aprovar as seguintes diretrizes e normas regulamentadoras de pesquisas envolvendo seres humanos. Recuperado em 10 dezembro, 2014, de http://conselho.saude.gov.br/resolucoes/2012/ Reso466.pdf.

Coutinho, M. P. L. (2005). Depressão infantil e representação social (2a ed.). João Pessoa, PB: Editora Universitária UFPB.

Ferigato, S. H., Campos, R. T. O., \& Ballarin, M. L. S. (2007). O atendimento a crise em saúde mental: ampliando conceitos. Revista de Psicologia da UNESP, 6(1), 31-44. Recuperado em 18 janeiro, 2015, de http://www2.assis.unesp.br/revpsico/index.php/revista/article/viewFile/ 44/84.

Foucault, M. (2010). Os anormais: curso no College de France (1974-1975). (2a ed.). São Paulo: Martins Fontes.

Franken, I., Coutinho, M. P. L., \& Ramos, M. N. P. (2012). Representações sociais, saúde mental e imigração internacional. Psicologia: Ciência e Profissão, 32(1), 202-219. Recuperado em 11 de março, 2016, de http://www.scielo.br/scielo.php?script=sci_arttext\&pid=S1414-989320120001000 =pt.

Gonçalves, R. W., Vieira, F. S., \& Delgado, P. G. G. (2012). Política de saúde mental no Brasil: evolução do gasto federal entre 2001 e 2009. Revista de Saúde Pública, 46(1), 51-58. Recuperado em 18 outubro, 2014, de http://www.scielo.br/pdf/rsp/v46n1/3113.pdf.

Hirdes, A. (2009). Autonomia e cidadania na reabilitação psicossocial: uma reflexão. Ciência \& Saúde Coletiva, 14(1), 165-171. Recuperado em 18 janeiro, 2015, de http://www.scielo.br/ pdf/csc/v14n1/a22v14n1.pdf.

Jodelet, D. (2001). As representações sociais. Rio de Janeiro: EdUERJ.

Jodelet, D. (2005). Experiência e representações sociais. In M. S. S. Menin \& A.M. Shimizu (Org.). Experiência e representação social: questões teóricas e metodológicas. São Paulo: Casa do Psicólogo.

Lopes, M. M. F. (2013). Trabalho e saúde mental: a inserção das pessoas com transtorno mental do Centro de Atenção Psicossocial de Rio das Ostras no mercado de trabalho: um diálogo possível. Trabalho de Conclusão de Curso de Graduação, Escola de Serviço Social, Departamento Interdisciplinar de Rio das Ostras, Universidade Federal Fluminense, Rio das Ostras, RJ. Recuperado em 18 janeiro, 2015, de http://www.puro.uff.br/tcc/2012-2/Magdalia\% 20Maria\%20Ferreira\%20Lopes.pdf.

Ministério da Saúde. Secretaria-Executiva. Secretaria de Atenção à Saúde (2005). Legislação em saúde mental: 1990-2004 (5a ed. ampl.). (Série E. Legislação de Saúde). Brasília: Ministério da Saúde. Recuperado em 18 dezembro, 2014, de http://bvsms.saude.gov.br/bvs/publicacoes/legislacao_mental.pdf.

Moscovici, S. (2001) Das representações coletivas às representações sociais. In D Jodelet. (Org.). Representações Sociais. Rio de Janeiro: Eduerj.

Moscovici, S. (2003). Representações Sociais. Investigações em psicologia social.Petrópolis, RJ: Vozes.

Nasi, C., \& Schneider, J. F. (2011). O Centro de Atenção Psicossocial no cotidiano dos seus usuários. Revista da Escola de Enfermagem da USP, 45(5), 1157-1163. Recuperado em 18 janeiro, 2015, de https://www.lume.ufrgs.br/bitstream/handle/10183/69735/000793185.pdf? sequence=1.

Nóbrega, S. M., \& Coutinho, M. P. L. (2011). O Teste de Associação Livre de Palavras. In M. P. L. Coutinho \& E. R. A. Saraiva (Orgs.). Métodos de pesquisa em Psicologia Social, perspectivas qualitativas e quantitativas (pp. 95-106). João Pessoa, PB: Editora Universitária.

Paulon, S., Neves, R., Dimenstein, M., Nardi, H., Bravo, O., Galvão, V. A. B. M., Severo, A. K. S., \& Figueró, R. (2013). A saúde mental no contexto da Estratégia Saúde da Família no Brasil. Psicologia para América Latina, (25), 24-42. Recuperado em 14 de março, 2016, de http://pepsic.bvsalud.org/scielo.php?script=sci_arttext\&pid=S1870-350X2013000200003\&Ing $=p t \& \operatorname{lng}=p t$. 
Pitta, A. M. F. (2011). Um balanço da reforma psiquiátrica brasileira: instituições, atores e políticas. Ciência \&Saúde Coletiva, 16(12), 4579-4589. Recuperado em 18 janeiro, 2015, de http://www.scielo.br/pdf/csc/v16n12/02.

Ribeiro, B. V. D. (2015). Pode o Louco Falar? Subjetividade, Saúde Mental e Mídia. In C. F. Braga \& J. A. F. Cirino (Orgs.) Representações sociais e comunicação: diálogos em construção (pp. 45 - 63). Goiânia: UFG/FIC/PPGCOM.

Rotelli, F. (2001). O inventário das subtrações. In F. Nicácio (Org.). Desinstitucionalização (pp. 61-64). (2a ed.). São Paulo: Hucitec.

Saraceno, B. (1999). Libertando identidades: da reabilitação psicossocial à cidadania possível. Rio de Janeiro: Instituto Franco Basaglia/ Te Corá.

Santos, N. S. et al. (2000). A autonomia do sujeito psicótico no contexto da reforma psiquiátrica brasileira. Psicologia: ciência e profissão, Brasília, 10(4), 46-53. Recuperado em 11 de março, 2015, de http://www.scielo.br/scielo.php?script=sci_\#end.

Venturini, E. (1999). Uma sociedade sem manicômio. Arquivos Brasileiros de Psiquiatria, Neurologia e Medicina Legal, 94(71), 13-18. 